\title{
Modelación y correlación entre series temporales de ciclos solares y series pluviométricas del extremo sur patagónico
}

\section{Modeling and correlation between time series of solar cycles and series of rainfall in the southern Patagonian extreme}

\author{
Tec. Oscar Bonfili ${ }^{1}$, oscar.bonfili@gmail.com \\ Mg. Dora Maglione², dmaglione@uarg.unpa.edu.ar \\ Prof. Julio Soto ${ }^{2}$, sotojulioa@ hotmail.com \\ ${ }^{1}$ Servicio Meteorológico Nacional, Oficina Meteorológica Río Gallegos \\ ${ }^{2}$ Instituto de Trabajo, Economía y Territorio - Universidad Nacional de la Patagonia Austral - \\ Unidad Académica Río Gallegos \\ Av. Gdor. Gregores y Piloto Rivero - Río Gallegos - Santa Cruz - Argentina
}

Recibido: 22/08/2018. Aceptado: 04/12/2018

\section{RESUMEN}

Hay estudios que avalan el hecho de que los cambios climáticos están relacionados con las variaciones que se producen en la emisión de la radiación solar. En particular, existe evidencia de que en ciertas regiones del planeta el número de manchas solares, la cual varía en ciclos de aproximadamente 11 años, influye sobre la precipitación, mientras que en otras regiones ese comportamiento no se verifica. Sin embargo, para la zona sur del continente americano no hay literatura que haya estudiado este comportamiento, por lo que en este trabajo se analizaron las series de precipitaciones para las ciudades de Río Gallegos (Argentina) entre 1928-2015 y para Punta Arenas (Chile) entre 1921-2015 con la finalidad de determinar la posible influencia de la cantidad de manchas solares sobre esta variable. Para llevar adelante este objetivo, en primer término, se modeló el comportamiento de las series usando al variograma para capturar la estructura de correlación temporal de los datos, y posteriormente se calcularon las correlaciones de las series de precipitaciones y las de manchas solares, y la de las precipitaciones y algunos rezagos de las manchas solares para poder determinar la posible asociación entre ambas variables. A partir de los resultados obtenidos del análisis se puede concluir que para esta región del planeta la cantidad de manchas solares no influye sobre la cantidad de lluvia caída.

Palabras clave: Manchas solares; Precipitación; Correlación; Series temporales

\begin{abstract}
There are studies that support the fact that climate changes are related to the variations that occur in the emission of solar radiation. In particular, there is evidence that in certain regions of the planet the number of sunspots, which vary in cycles of approximately 11 years, influences rainfall, while in other regions that behavior does not occur. However, for the southern area of the American continent there is no literature that has studied this behavior, so in this work the rainfall series for the cities of Río Gallegos (Argentina) between 1928-2015 and Punta Arenas (Chile) between 1921-2015 were analyzed in order to determine the possible influence of the amount of sunspots over this variable. In order to carry out this
\end{abstract}


objective, the behavior of the series was modeled first using the variogram to capture the temporal correlation structure of the data, and subsequently the correlations of the series of precipitations and those of sunspots were calculated, and that of the precipitations and some lags of the sunspots to be able to determine the possible association between both variables. From the results obtained from the analysis it can be concluded that for this region of the planet the amount of sunspots does not influence the amount of rainfall.

Key words: Sunspots; Precipitation; Correlation; Temporal series

\section{INTRODUCCIÓN}

En la literatura no se han encontrado estudios que analicen la correlación en latitudes mediaaltas del hemisferio sur, como lo es el extremo austral patagónico, entre las series de precipitaciones y la cantidad de manchas solares.

Conseguir series de precipitación lo suficientemente largas, depuradas a fin de evitar incosistencias producto de cambios de emplazamientos, cambio de instrumentación, etc., es una tarea poco fácil. Las series más completas en la región, resultaron ser las de la ciudad de Río Gallegos (Argentina) y Punta Arenas (Chile), aún cuando los emplazamientos de ambas estaciones meteorológicas han variado a lo largo del extenso tiempo considerado (19212015). Como morfológicamente la geografía es homogénea, no se ha considerado corregir los valores de precipitación a lo largo de la serie.

Así que, con el fin de determinar esta posible influencia, se consideraron las series pluviométricas de dichas ciudades, Río Gallegos y Punta Arenas, ambas ubicadas en el extremo sur del continente americano (ver Fig.1). A partir de esta información se modeló el comportamiento de las series usando al variograma para capturar la estructura de correlación temporal de los datos, y además se calcularon las correlaciones de las series de precipitaciones y las de manchas solares, la de las precipitaciones y algunos rezagos de las manchas solares para poder determinar la posible asociación entre ambas variables.

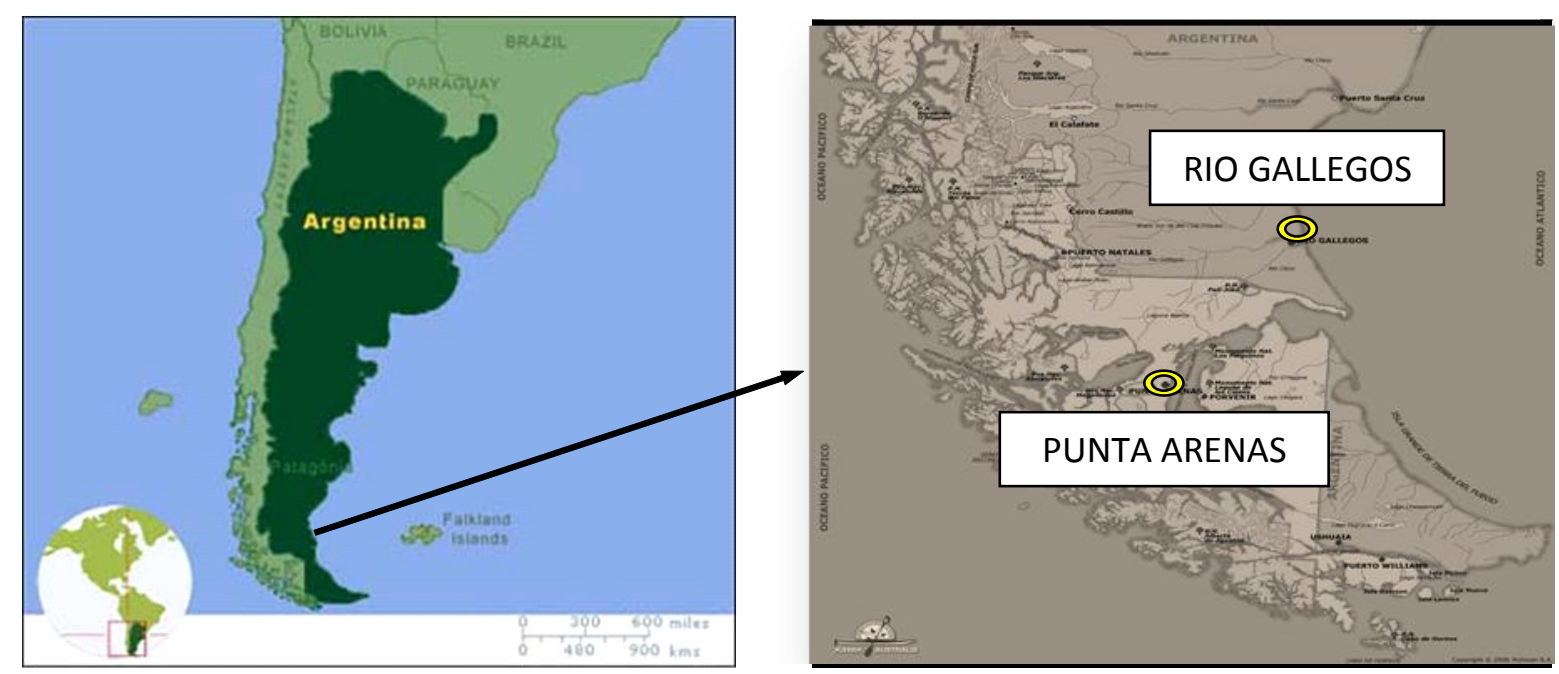

Figura 1: Ubicación de las ciudades de Río Gallegos y Punta Arenas

Las geoformas que predominan en Punta Arenas coinciden con la "unidad morfológica de suaves ondulaciones y pequeños cordones de escasa altura, que corresponden a depósitos morrenicos que encierran numerosas cuencas lacustres" Arenas Vásquez; F. et al. (2010). 
La región que enmarca a ambas ciudades se caracteriza por un clima semiárido caracterizado por las bajas precipitaciones anuales asociado a la dinámica de los procesos meteorológicos en contacto con los factores geográficos.

La ubicación para Río Gallegos y Punta Arenas, ambas a escasos kilómetros del Mar Argentino y del Estrecho de Magallanes respectivamente, haría pensar en un clima eminentemente marítimo, con elevado índice de humedad y escasa amplitud térmica anual. Sin embargo, uno de los aspectos climáticos que caracteriza a este espacio es el predominio de un ambiente semiárido continental producto de los constantes vientos del Oeste, escasas precipitaciones (aunque abundante nubosidad) durante todo el año, una notoria amplitud térmica diaria, y una insolación media anual escasa (Bonfili, Soto y Maglione, 2016; Maglione et al. 2014). Las precipitaciones, particularmente, presentan un promedio histórico de 252 mm (1928/2015) para Río Gallegos y de 400 mm (1921/2015) para Punta Arenas. Los valores extremos que presenta Río Gallegos son de $416.5 \mathrm{~mm}$ (2002) y $127.3 \mathrm{~mm}$ (1968), así como para Punta Arenas son $797.0 \mathrm{~mm}$ (1950) y $216.6 \mathrm{~mm}$ (1966), considerando los periodos trabajados en este estudio.

Para este análisis se empleó una serie de precipitaciones mensuales y anuales de ochenta y ocho años (1928-2015), pertenecientes a la estación Río Gallegos del Servicio Meteorológico Nacional (5138' S; 69 ${ }^{\circ} 17^{\prime} \mathrm{O}$ y altitud: 19 m s.n.m.), y otra de noventa y cinco años (19212015) pertenecientes a la estación Punta Arenas de la Dirección Meteorológica de Chile

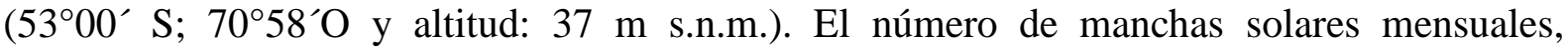
correspondientes a los ciclos solares 16 al 24 (1921-2015), se obtuvo de los datos publicados por WDC-SILSO, Observatorio Real de Bélgica (Royal Observatory of Belgium, Brussels).

Este trabajo está organizado de la siguiente manera, en la siguiente sección se describe el comportamiento de las manchas solares. Posteriormente, se encuentra la metodología utilizada y los resultados del análisis. Y finalmente en la última sección se presentan algunas conclusiones.

\section{MARCO TEORICO}

Nuestra estrella madre es dadora de vida y condicionante de toda nuestra existencia. A simple vista semeja un simple cuerpo de brillo constante, sin mayores características sobresalientes. La realidad es muy diferente, ya que en su superficie ocurren acontecimientos violentos donde se liberan enormes cantidades de energía que, viajando por el espacio, pueden $-\mathrm{y}$ de hecho lo hacen- afectar el entorno terrestre: fulguraciones, emisiones de masa coronal, prominencias, manchas, etc.

Respecto a las manchas solares (MS), se pude describir como regiones de la superficie solar con una temperatura mucho más baja que su entorno, y con una actividad magnética muy intensa. Estas manchas duran en general unas pocas semanas, pero el periodo de ocurrencia varía en ciclos de 131+14 meses, de mínimo a mínimo, (aproximadamente 11 años).

Asociadas a las manchas también ocurren las explosiones solares, las cuales lanzan grandes cantidades de material hacia las afueras del sol, formando parte del viento solar y alcanzando a la tierra, causando las tormentas magnéticas y las auroras boreales y australes.

Existe una relación entre el número variable de manchas solares (SSN) y la intensidad del flujo de radiación solar que incide sobre la Tierra. En un máximo de actividad solar el sol irradia, en promedio, una mayor cantidad de energía que en períodos de mínima actividad. Con el número de manchas solares aumenta proporcionalmente el número de tormentas solares (en la Fig.2 se observan imágenes de manchas solares). Al llegar a la Tierra, este gas 
choca contra el campo magnético de nuestro planeta. El viento solar aumenta en períodos de máxima actividad, alcanzando las capas altas de la atmósfera terrestre e influyendo sobre el campo magnético de la tierra, como ya se ha mencionado.

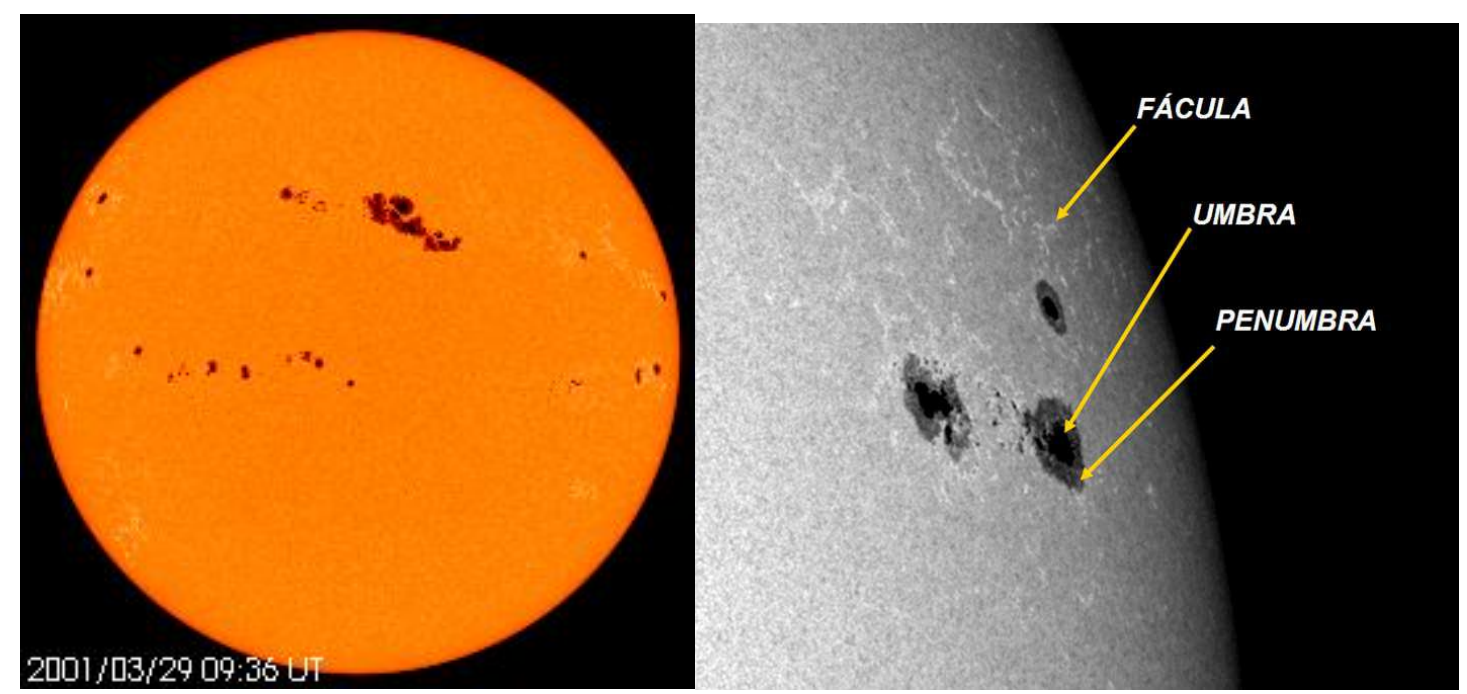

Figura 2: Manchas solares de tamaño inusual. Parece contradictorio, que la actividad solar aumente a causa de las manchas oscuras del sol. Pero alrededor de estas manchas la intensidad de la radiación es más alta de lo normal. Fuente: SOHO project / NASA

Los ciclos de actividad solar, van a influir directa o indirectamente en el comportamiento de fenómenos climáticos terrestres, como la cantidad de lluvia caída en nuestro planeta a nivel global, o la temperatura superficial promedio de la tierra (Pedreros, 2013). Esto puede observarse en la correlación existente entre el grosor de los anillos de los árboles y el ciclo de actividad magnética del sol. El grosor de los anillos indica mejores condiciones de crecimiento del árbol en el año respectivo, lo cual, entre otras cosas, es un apropiado proxi como indicador de abundancia de lluvias y/o altas temperaturas (Jiménez, 2012), como pueden serlo las capas glaciarias, los sedimentos marinos o las formaciones de Elatina.

En la literatura científica mundial existen cantidad de artículos que dan cuenta de zonas con influencia del ciclo de las manchas solares en la precipitación y de lugares en los que no se detecta esta relación. Por ejemplo, Bal y Bose (2010) encuentran que la precipitación y el número de manchas solares en las series temporales que analizan no están correlacionadas anualmente, ni estacionalmente con localizaciones de Australia, Canadá, Etiopía, Groenlandia, India, Islandia, Japón y Sudáfrica, pero sí con los puntos estudiados para el Reino Unido, Estados Unidos y Rusia con un nivel de confianza del 95\%. Por su parte, Zhao, Wang y Zhao (2012) encuentran que considerando China a nivel nación, la precipitación no está correlacionada con la cantidad de manchas solares, sin embargo, en muchas regiones, la variabilidad de la precipitación estival en un periodo de más de 8 años se correlaciona significativamente con esta variable (a un nivel de confianza mayor al 95\%). También Hiremath, Manjunath y Soon (2015) avanzan en el estudio sobre la evidencia estadística de que la actividad solar influye en las lluvias del monzón de verano en la India. Además, se observan correlaciones altas entre el número de manchas solares y el flujo del Río Paraná (Mauas, Buccino y Flamenco 2010); correlaciones entre el ciclo solar y fuertes lluvias en el este de África, ocurridas en el siglo XX (Stager et al., 2007); correlaciones de significación moderada a elevada en Tamil Nadu (India) (Selavaraj y Adita, 2012); correlaciones significativas entre la pluviometría ibérica y el periodo solar más activo registrado (19391969), con un desfase de tres a cuatro años (Romeu i Codina y López-Bustin, 2006; Clavet et al., 2001); una relación positiva entre los años de máxima actividad solar y la aparición de 
eventos extremos en las precipitaciones, en el sector rural y urbano de Azul-Argentina (Navarro, Vilatte y Aguas, 2013).

Volviendo al hilo conductor de relaciones: a mayor actividad magnética del sol, mayor es la producción de viento solar. A su vez el viento solar altera el campo magnético terrestre e impide la llegada normal de rayos cósmicos (galácticos y solares) a la tierra, lo que incide en una menor producción de carbono-14 $\left({ }^{14} \mathrm{C}\right)$. Existen evidencias científicas de que los rayos cósmicos galácticos (RCG) son una fuente extraplanetaria de energía que también influencia el clima de la Tierra (Svensmark y Friis-Christensen 1997; Carslaw, Harrison y Kirkby, 2002). El carbono-14 (radioisótopo cosmogénico) se incorpora al material orgánico, lo que permite un seguimiento de su abundancia en el tiempo. En la Tabla 1 se presentan algunos periodos extremos de actividad solar en función de la concentración ${ }^{14} \mathrm{C}$ en los anillos de los árboles.

Tabla 1: Algunos periodos de máxima y mínima actividad solar en el pasado a partir de la concentración de ${ }^{14} \mathrm{C}$ en los anillos de los árboles

\begin{tabular}{ll}
\hline Evento & Duración aproximada \\
\hline $\begin{array}{l}\text { Máximo sumerio } \\
\text { Máximo piramidal }\end{array}$ & $2720-2610$ a.C \\
Máximo de & 1870-17060 a.C \\
Stonehenge & a.C \\
Mínimo egipcio & $1420-1260$ a.C \\
Mínimo homérico & $820-640$ a.C \\
Mínimo griego & $440-360$ a.C \\
Máximo romano & 20a.c. -80 d.C. \\
Mínimo medieval & $640-710$ d.C. \\
Máximo medieval & $1120-1280$ d.C. \\
Mínimo de Spoerer & 1400-1510 d.C. \\
Mínimo de Maunder & $1640-1710$ d.C. \\
Máximo moderno & empezó en 1800 \\
\end{tabular}

Los resultados que arroja el estudio de depósitos de Be10 son muy similares a los obtenidos del estudio del C14.

Si bien la contribución de los ciclos solares al balance de energía en la baja troposfera no es significativa en el aumento de la energía recibida desde el sol (0,1\%) (Abbot, 1966; Legras Mestre, Bard y Yiou, 2010), hay pruebas crecientes de que la actividad solar tiene una influencia en el clima terrestre (Hathaway y Wilson, 2004). Se ha comprobado una conexión entre la estratosfera y la troposfera, como influencia de los ciclos solares a través de la interacción del ozono y las anomalías en la temperatura y el viento (Baldwin y Dunkerton, 2005). La relación entre los ciclos solares y el balance de energía en superficie se explica por el efecto que puede tener la ionización solar sobre la formación de nubes bajas en nuestra 
atmósfera, incrementando la contra-radiación atmosférica (Lane, Nichols y Osborn, 1994; Pallé, Butler y O' Brien, 2004; Svensmark, 1998; Tinsley y Yu, 2004).

Es de particular importancia la radiación solar en el ultravioleta extremo (UVE), la cual alcanza su punto de mayor intensidad durante los años cercanos al máximo solar. Esto puede afectar considerablemente la química y la estructura térmica de la atmósfera superior. Hay muchos caminos "de arriba hacia abajo" para que el Sol ejerza su influencia. Por ejemplo (según Jackman en National Research Council, 2012) el óxido nitroso (NOx), creado por partículas energéticas solares (SEP) y rayos cósmicos en la estratósfera, puede reducir los niveles de ozono en varios puntos porcentuales. Debido a que el ozono absorbe la radiación UV, tener menos ozono implica que más rayos UV del Sol pueden llegar a la superficie de la Tierra. El enfriamiento de la estratósfera polar asociado con la pérdida de ozono, incrementa el gradiente horizontal de temperatura cerca de la tropopausa, alterando el flujo de momento angular en los vórtices de latitudes intermedias (Held, 2012). El momento angular es importante ya que el equilibrio del mismo en la tropósfera, controla los vientos superficiales que se mueven hacia el Oeste ('westerlies'). En otras palabras: el efecto de la actividad solar en la atmósfera superior puede, a través de una complicada cadena de influencias, empujar a las tormentas que se encuentran en la superficie fuera de su curso natural. El calentamiento de la estratosfera parece debilitar el cinturón subtropical de altas presiones y, en consecuencia, el flujo de vientos del oeste, con lo que disminuye la lluvia en la fachada oceánica de los continentes de zonas templadas. Este debilitamiento permite el reforzamiento de circulaciones meridianas de $\mathrm{N}$ a $\mathrm{S}$ o de $\mathrm{S}$ a $\mathrm{N}$, con ingresos fríos o cálidos asociados a anticiclones de bloqueo de larga duración.

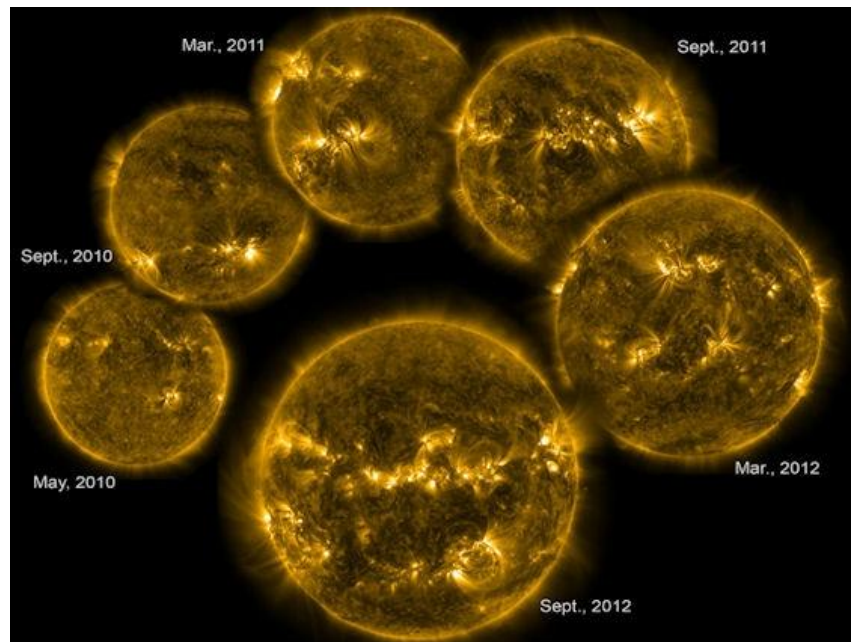

Figura 3: Estas seis imágenes del Sol en el ultravioleta extremo, captadas por el Observatorio de Dinámica Solar (Solar Dynamics Observatory o SDO), de la NASA, registran el creciente nivel de la actividad solar conforme el Sol se acerca hacia la cúspide del ciclo de manchas solares de 11 años de duración

Así, la variabilidad solar estaría produciendo un efecto sobre el clima, especialmente en el Pacífico. Así, cuando se analizan los datos correspondientes a la temperatura superficial del océano durante los años en que hay más manchas solares, el Pacífico tropical muestra un pronunciado patrón similar a "La Niña". Además, "hay indicios de incrementos de precipitación en la ZITC (Zona Inter-Tropical de Convergencia) del Pacífico y en la ZCPS (Zona de Convergencia del Pacífico Sur), así como de presiones a nivel del mar que están por encima de lo normal en latitudes intermedias del Pacífico Norte y Sur", las cuales se correlacionan con los picos del ciclo de manchas solares (Meehl y Teng, 2012). 
Cuando el equilibrio radiativo de la Tierra es alterado, como ocurre cuando hay un cambio en la influencia producida por el ciclo solar, no todos los lugares se ven afectados de la misma forma. El Pacífico ecuatorial central generalmente se torna más frío, la escorrentía de ríos en Perú se ve reducida y las condiciones en el oeste de Estados Unidos se vuelven más secas (según Amman en National Research Council, 2012).

La actividad solar (AS) afecta el clima debido a la variabilidad de baja frecuencia repetida cada 11 años (ciclo de Schwabe), 22 años (ciclo de Hale), 30-33 años (ciclo de Brückner) y de 80-100 años (ciclo de Gleissberg) (Lean, Beer y Bradley, 1995; Raspopov, Dergachevb y Kolstro, 2004; Kasatkina et al. 2007); en la Fig.3 se puede observar la actividad creciente del ciclo de manchas solares al acercarse al máximo del ciclo y en la Fig. 4 la estimación del número de manchas solares en los últimos 400 años. Las manchas solares (MS) son un registro directo de la AS: A mayor número de MS, mayor es la radiación emitida por el Sol (Usoskin et al., 2003). La AS es responsable de modular el flujo de rayos cósmicos galácticos (RCG) que ionizan la atmósfera de la Tierra y produce el 14C (Oeschger, Beer y Andree, 1987); cuando el Sol es menos activo, el flujo de RCG es mayor sobre la Tierra aumentando el contenido de 14CO2 en la atmósfera, produciendo una mayor cobertura global de nubes (Svensmark y Friis-Christensen, 1997; Kniveton, 2004; Todd y Kniventon, 2004; Harrison y Stepheson, 2006; Svensmark , Bondo y Svensmark, 2009). El clima medio de la tierra depende de la energía proveniente del espacio exterior, mientras que la composición de gases atmosféricos y vapor de agua determinan en qué medida ésta se absorbe o se emite (Yepes y Bukeridge, 2011).

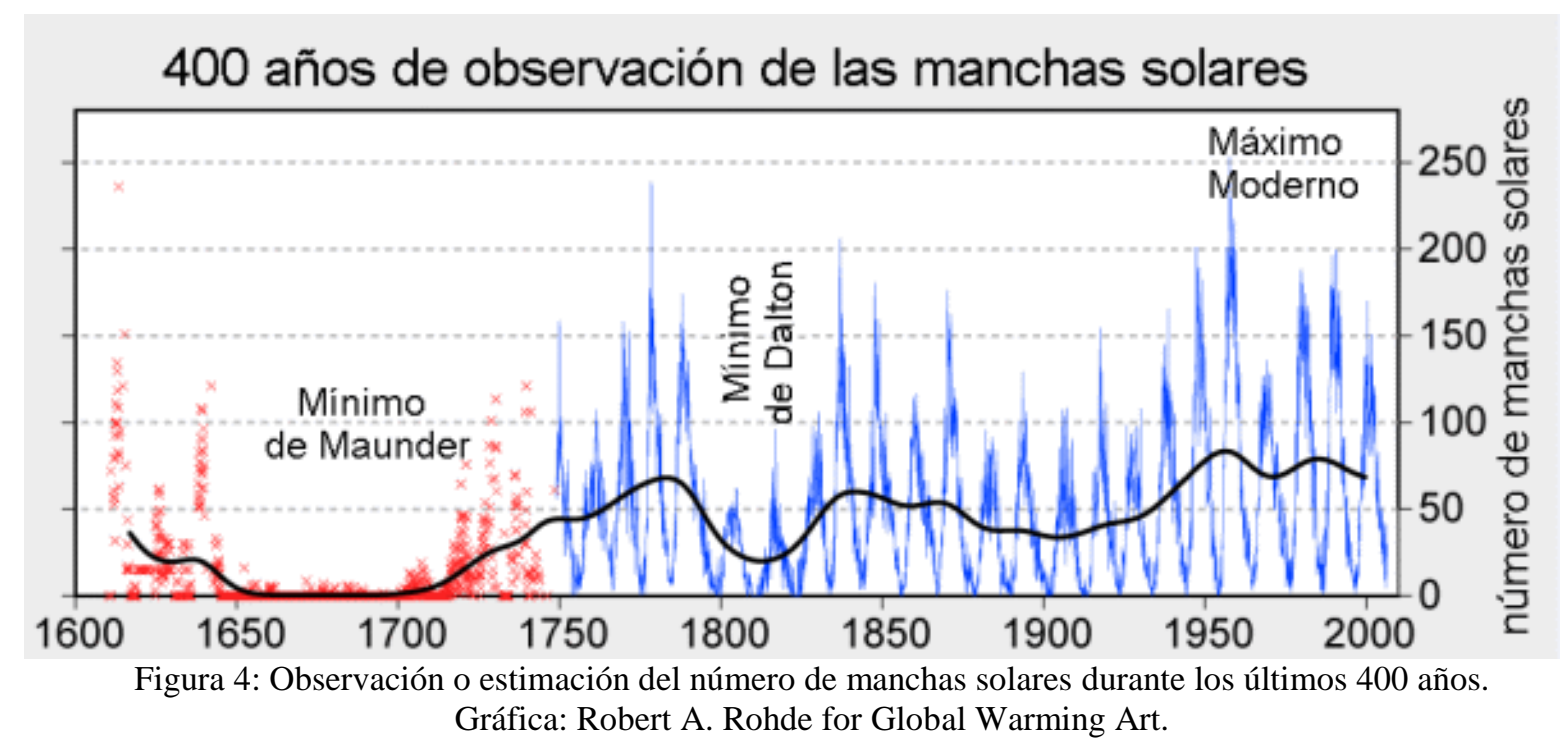

\section{METODOLOGIA Y RESULTADOS}

La metodología utilizada se basa en la modelación de las series temporales de precipitaciones y manchas solares, usando al variograma como función que modela la estructura de correlación temporal de las mismas. Una vez obtenidos los modelos, se calcularon distintas medidas de correlaciones entre las series de precipitaciones de ambas ciudades y la serie de las manchas solares y de algunos de los rezagos de esta última, para poder cuantificar la asociación entre la variable precipitación y el número de manchas solares.

Los análisis estadísticos que se presentan a continuación se realizaron con el software libre $\mathrm{R}$ (https://www.r-project.org/). De este programa se utilizaron las funciones básicas y los 
paquetes geoR y gstat para la modelación espacio-temporal de datos geoestadísticos, y los paquetes lattice y FBasics para el manejo de gráficos y funciones estadísticas más avanzadas.

Los datos de precipitación de Río Gallegos se obtuvieron del Servicio Meteorológico Nacional (https://www.smn.gob.ar/) y los de la estación de Punta Arenas de la Dirección Meteorológica de Chile (http://www.meteochile.gob.cl/PortalDMC-web/index.xhtml). El número de manchas solares mensuales corresponden a datos publicados por el Observatorio Real de Bélgica (https://www.astro.oma.be/en/information/library-observatory-rmi/).

\subsection{Modelación de las series mensuales}

Las series temporales de la variable precipitación para ambas estaciones y la del número de manchas solares presentan correlación en el tiempo (en todos los casos el p-valor para el índice de Morán es mucho menor a 0.0001), así que para modelar las series es necesario incorporar la dependencia temporal existente. Para modelar la estructura de correlación temporal de las distintas series utilizamos el semivariograma experimental calculado con los datos de cada una de las series temporales, y luego se ajustaron diferentes modelos teóricos (exponencial, esférico y gaussiano) y se escogió el de menor varianza en el error.

En el caso de la precipitación mensual, los datos no siguen una distribución normal, por lo que fue necesario realizar una transformación y se modeló la estructura de correlación del logaritmo de la serie desplazada. El modelo aditivo correspondiente para la variable transformada, se convierte en un modelo multiplicativo cuando la variable es regresada a la escala original.

Para la ciudad de Río Gallegos y para el logaritmo de la precipitación media mensual, el modelo que mejor ajustó la estructura de correlación temporal fue el modelo exponencial.

$$
\gamma(h)=\left\{\begin{array}{c}
0.0361 \quad h=0 \\
0.00361+0.4075\left(1-e^{-\frac{h}{0.6431}}\right) \text { sino }
\end{array}\right.
$$

Con lo cual, los datos (expresados en la escala original) pueden modelarse por medio del modelo:

$$
Z(t)=-4+20.2853 \cdot e^{\varepsilon(t)}
$$

Donde $\mathrm{Z}$ es la precipitación media mensual en el mes t y la variación a pequeña escala sigue una distribución normal con estructura de correlación dada el semivariograma anterior.

El coeficiente de variazación estructural en este caso es de $100 \%$ por lo que no se puede ignorar la correlación espacial al realizar predicciones.

En el caso de la cuidad de Punta Arenas, el modelo resultante es:

$$
Z(t)=-6+33.7574 . e^{\varepsilon(t)}
$$

Donde $\mathrm{Z}$ es la precipitación media mensual en el mes t y la variación a pequeña escala sigue una distribución normal con estructura de correlación dada por

$$
\gamma(h)=\left\{\begin{array}{c}
0.2549 \quad h=0 \\
0.2549+0.0723\left(1-e^{-\frac{h}{2.9088}}\right) \text { sino }
\end{array}\right.
$$

Donde nuevamente el modelo exponencial es el que mejor ajusta al semivariograma experimental. 
En el caso de las manchas solares, no es necesario aplicar una transformación a los datos, por lo que la serie es modelada por un modelo aditivo. El mismo resulta ser

$$
\mathrm{Z}(\mathrm{t})=96.97+\varepsilon(\mathrm{t})
$$

Donde $\mathrm{Z}$ es la cantidad media mensual de manchas solares en el mes $\mathrm{t}$ y la variación a pequeña escala sigue una distribución normal con estructura de correlación modelada nuevamente por un semivariograma de la familia exponencial dado por:

$$
\gamma(h)=\left\{\begin{array}{c}
269.32 h=0 \\
269.32+5106.33\left(1-e^{-\frac{h}{43.90}}\right) \text { sino }
\end{array}\right.
$$

En la Fig. 5 se muestran los distintos modelos para la estructura de correlación temporal de las series mensuales analizadas.
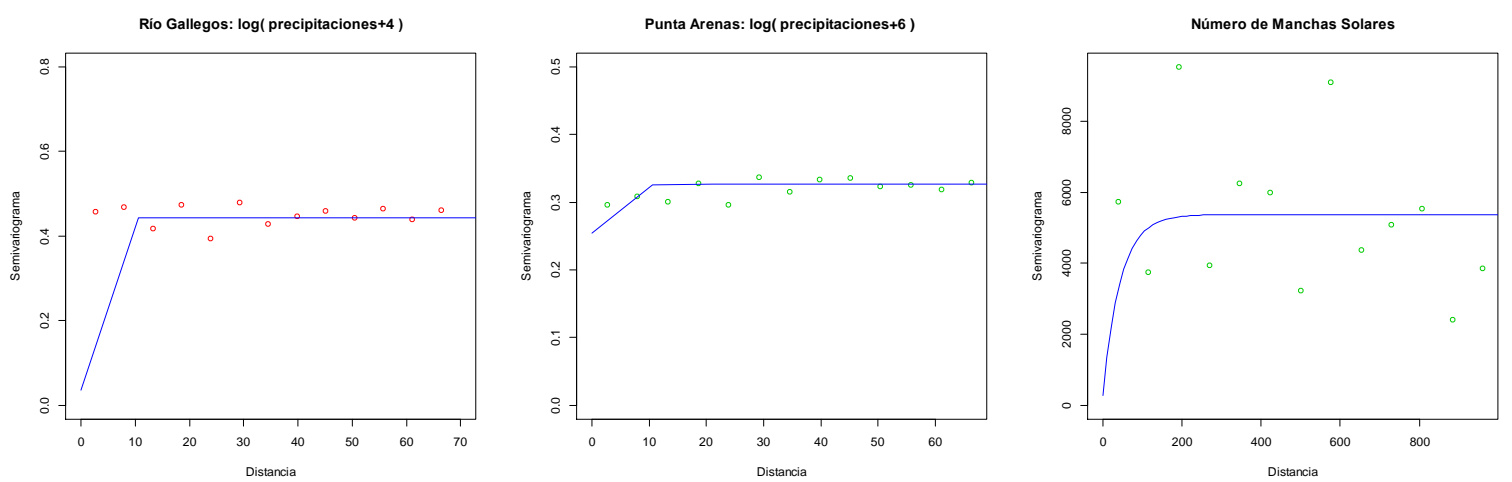

Figura 5: Semivariogramas de las series de precipitaciones mensuales transformadas de Río Gallegos y Punta Arenas y para el número de manchas solares

\subsection{Modelación de las series anuales}

Las series temporales de la variable precipitación para ambas estaciones presentan correlación temporal (el p-valor para el índice de Morán en la ciudad de Río Gallegos es de 0.0003 y el correspondiente a la ciudad de Punta Arenas es 0.0001). Estas distribuciones son normales, por lo que no es necesario aplicar transformaciones a las series temporales.

La estructura de correlación temporal para la precipitación utilizando el semivariograma para Río Gallegos y Punta Arenas puede ser modelada por las funciones:

$$
\begin{gathered}
\gamma(h)=\left\{\begin{array}{c}
17.325 \quad h=0 \\
17.3254+6.215\left(1-e^{-\frac{h}{5.415}}\right) \text { sino }
\end{array}\right. \\
\gamma(h)=\left\{\begin{array}{c}
36.397 \quad h=0 \\
36.397+48.010\left(1.5 \frac{h}{3.987}-0.5\left(\frac{h}{3.987}\right)^{3}\right) \text { sino }
\end{array}\right.
\end{gathered}
$$

En este caso el modelo teórico usado para los datos de la ciudad de Río Gallegos es nuevamente el exponencial, pero para la ciudad de Punta Arenas el que posee menor varianza es uno proveniente de la familia esférica.

Por lo que el modelo structural estructural para la precipitación media mensual puede ser modelada por:

$Z(t)=21.094+e(t)$, en el caso de Río Gallegos

$\mathrm{Z}(\mathrm{t})=33.358+\mathrm{e}(\mathrm{t})$, en el caso de Punta Arenas 
La serie de las manchas solares anuales queda modelada como $Z(t)=96.898+e(t)$ donde la estructura de correlación temporal es capturada por un variograma de la familia esférica dado por

$$
\gamma(h)=\left\{\begin{array}{c}
0 \text { si } h=0 \\
3166.779\left(1.5 \frac{h}{6.186}-0.5\left(\frac{h}{6.186}\right)^{3}\right) \quad \text { sino }
\end{array}\right.
$$

En la Fig. 6 se muestran los variogramas para las series de precipitaciones anuales de ambas ciudades.
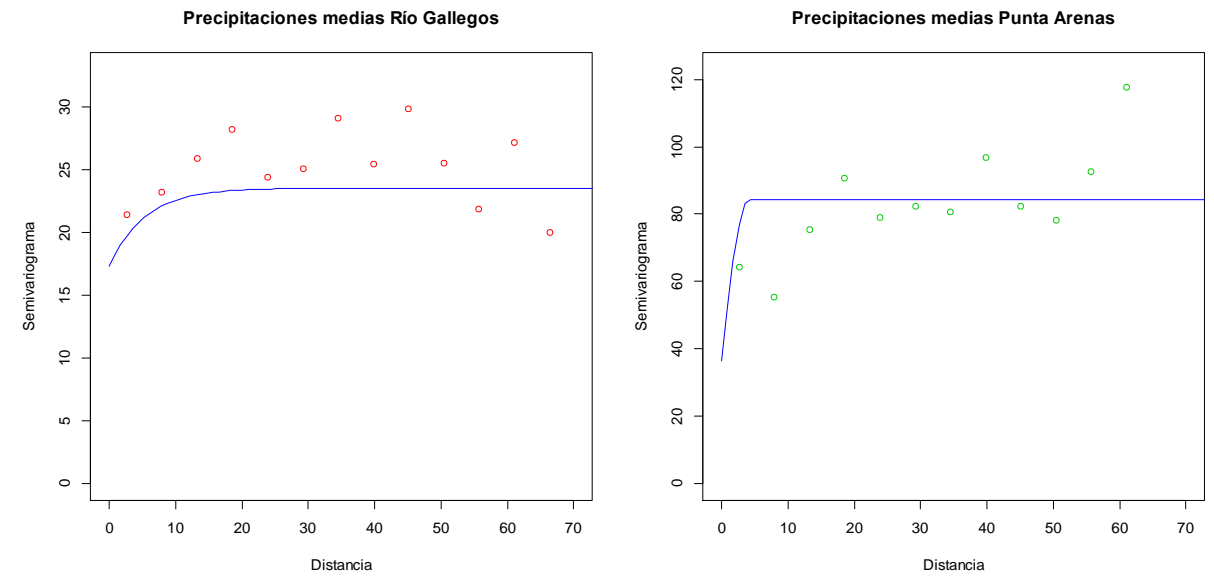

Figura 6: Semivariogramas de las series de precipitaciones anuales de Río Gallegos y Punta Arenas

Por otro lado, en la Fig. 7 se representan temporalmente las series anuales de precipitaciones para ambas ciudades y la de la cantidad de manchas solares en el periodo 1928-2015.

Series para las medias anuales (1928-2015)

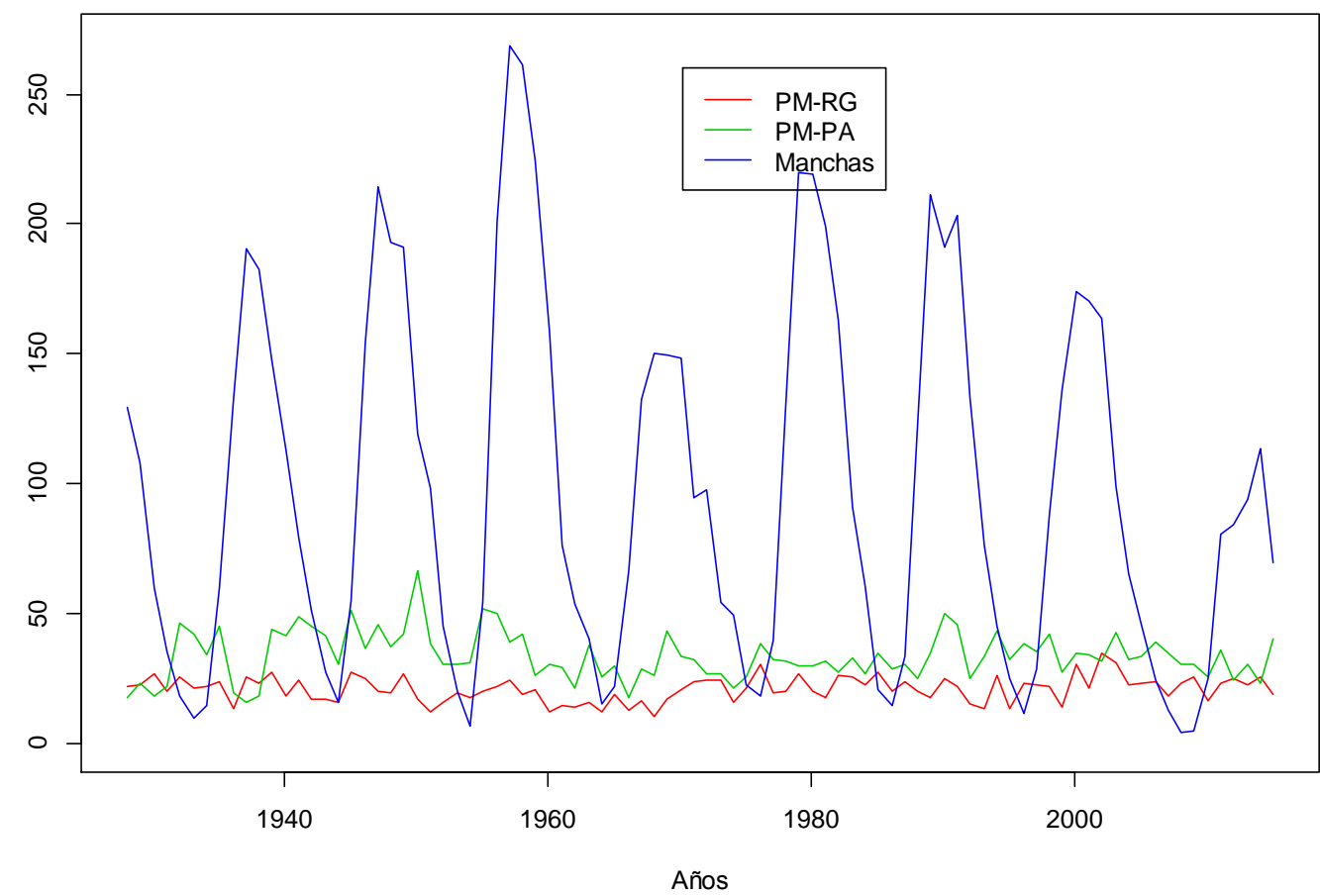

Figura 7: Series de precipitaciones anuales consideradas en el período 1928 a 2015 para Río Gallegos y Punta Arenas, y el correspondiente del número de manchas solares 


\subsection{Correlaciones entre las series}

La intención detrás de este trabajo es intentar dilucidar si existe asociación entre la cantidad de manchas solares y la cantidad de lluvia caída, por lo que estudiamos el coeficiente de correlación.

Así, si $\rho$ representa al coeficiente de correlación entre la serie de la cantidad de manchas solares y la precipitación en una localidad, bajo el supuesto de que el número de manchas solares influye sobre la precipitación se planteó la siguiente hipótesis nula (junto a su alternativa):

$$
\begin{aligned}
& \left.H_{0}\right) \rho=0 \\
& \left.H_{1}\right) \rho \neq 0
\end{aligned}
$$

Como estadístico de prueba para poder dar respuesta a esta hipótesis se calculó el coeficiente de Pearson (o el de Spearman cuando las series no siguen una distribución normal) junto con el p-valor para decidir si aceptar o rechazar la hipótesis nula planteada.

Otra hipótesis de trabajo es que la influencia de cantidad de manchas solares se ve reflejada en la precipitación de periodos subsiguientes, por lo que se plantearon las hipótesis:

$$
\begin{aligned}
& \left.H_{0}\right) \rho_{K}=0 \\
& \left.H_{1}\right) \rho_{K} \neq 0
\end{aligned}
$$

Donde $\rho_{\mathrm{K}}$ es la correlación entre el número de manchas solares y la cantidad de precipitaciones $\mathrm{K}$ periodos posteriores, $\mathrm{K}=1,2,3, \ldots$ Nuevamente para dar respuesta a estos interrogantes se calcularon los coeficientes y p-valor de la serie de manchas solares con las de precipitaciones desplazadas en $\mathrm{K}$ periodos.

En la Tabla 2 se presentan los coeficientes de correlación y el p-valor para las series y para los primeros tres desplazamientos para las series mensuales y la Tabla 3 para las series anuales. En las mismas también se exhiben las correlaciones entre las precipitaciones de ambas

\begin{tabular}{|c|c|c|c|c|c|c|c|}
\hline \multirow{2}{*}{\multicolumn{3}{|c|}{ SERIES MENSUALES }} & \multirow{3}{*}{$\begin{array}{l}\text { Precipitac. } \\
\text { anuales } \\
\text { Punta } \\
\text { Arenas } \\
0.045\end{array}$} & \multirow{2}{*}{\multicolumn{4}{|c|}{ Manchas solares con rezagos }} \\
\hline & & & & & & & \\
\hline \multirow{4}{*}{$\begin{array}{l}\text { Río } \\
\text { Gallegos }\end{array}$} & \multirow{2}{*}{ Pearson } & Coefic. & & 0.0069 & 0.0159 & 0.0107 & 0.0112 \\
\hline & & p-valor & 0.0000 & 0.8231 & 0.6063 & 0.7292 & 0.7161 \\
\hline & \multirow[t]{2}{*}{ Spearman } & Coefic. & 0.4179 & -0.0022 & 0.0090 & 0.0012 & 0.0009 \\
\hline & & p-valor & 0.0000 & 0.9429 & 0.7703 & 0.9682 & 0.9774 \\
\hline \multirow{4}{*}{$\begin{array}{l}\text { Punta } \\
\text { Arenas }\end{array}$} & \multirow[t]{2}{*}{ Pearson } & Coefic. & & 0.0337 & 0.0216 & 0.0198 & 0.0152 \\
\hline & & p-valor & & 0.2739 & 0.4830 & 0.5207 & 0.6217 \\
\hline & \multirow[t]{2}{*}{ Spearman } & Coefic. & & 0.0043 & -0.0053 & -0.0106 & -0.0138 \\
\hline & & p-valor & & 0.8902 & 0.8631 & 0.7305 & 0.6554 \\
\hline
\end{tabular}
localidades junto a sus p-valores, tanto para las series mensuales como para las anuales.

Tabla 2: Coeficientes de correlación de Pearson y Spearman con sus p-valores para las series mensuales. Los valores de $\mathrm{k}$ indican la cantidad de periodos considerados en relación al actual

En la Tabla 2, se observa que existe una asociación moderada entre las precipitaciones mensuales de ambas ciudades ( 0.4245 y 0.4179 para los coeficientes de Pearson y Spearman respectivamente). Por su parte las columnas correspondientes a manchas solares indican que 
no existe asociación entres la cantidad de manchas solares mensuales y las precipitaciones mensuales del mes en que se producen las mismas $(\mathrm{k}=0)$, ni de los tres meses subsiguientes $(\mathrm{k}=1, \mathrm{k}=2, \mathrm{k}=3)$ en ninguna de las dos ciudades con una confianza del 95\%.

En la Tabla 3, se contempla que existe una asociación débil entre las precipitaciones anuales de ambas ciudades ( 0.1772 y 0.2048 para los coeficientes de Pearson y Spearman respectivamente). Por su parte las columnas correspondientes a manchas solares indican que no existe asociación entre la cantidad de manchas solares anuales y las precipitaciones anuales del año en que se producen las mismas $(\mathrm{k}=0)$, ni con las precipitaciones anuales del año siguiente $(\mathrm{k}=1)$, o con el de dos años posteriores $(\mathrm{k}=2)$, ni a tres años vista $(\mathrm{k}=3)$ en ninguna de las dos ciudades, lo cual puede afirmarse con una confianza del $95 \%$ ya que todos los $\mathrm{p}$ valores son superiores a 0.05 .

Tabla 3: Coeficientes de correlación de Pearson y Spearman con sus p-valores para las series anuales. Los valores de $\mathrm{k}$ indican la cantidad de periodos considerados en relación al actual

\begin{tabular}{|l|l|l|l|l|l|l|l|}
\hline SERIES ANUALES & & $\begin{array}{l}\text { Precipitac. } \\
\text { Anuales }\end{array}$ & \multicolumn{3}{c|}{ Manchas solares con rezagos } \\
\cline { 4 - 8 } & & $\begin{array}{l}\text { Punta } \\
\text { Arenas }\end{array}$ & $\mathrm{K}=0$ & $\mathrm{~K}=1$ & $\mathrm{~K}=2$ & $\mathrm{~K}=3$ \\
\hline $\begin{array}{l}\text { Río } \\
\text { Gallegos }\end{array}$ & Pearson & Coefic. & 0.1772 & 0.0959 & 0.0538 & -0.0161 & -0.1002 \\
\cline { 3 - 8 } & p-valor & 0.0986 & 0.3740 & 0.6294 & 0.8829 & 0.3615 \\
\cline { 3 - 8 } & Spearman & Coefic. & 0.2048 & 0.0834 & 0.0527 & -0.0003 & -0.0605 \\
\hline & p-valor & 0.0556 & 0.4398 & 0.6279 & 0.9978 & 0.5823 \\
\hline \multirow{2}{*}{$\begin{array}{l}\text { Punta } \\
\text { Arenas }\end{array}$} & Pearson & Coefic. & & 0.0669 & 0.0767 & 0.0680 & 0.0566 \\
\cline { 3 - 8 } & Spearman & p-valor & & 0.5360 & 0.4802 & 0.5337 & 0.6069 \\
\hline & Coefic. & & 0.0408 & 0.0285 & 0.0447 & 0.0479 \\
\hline
\end{tabular}

\section{CONCLUSIONES}

En este trabajo se han modelado las series de precipitación tanto de los datos mensuales y anuales para las ciudades de Río Gallegos y de Punta Arenas, al igual que para la cantidad de manchas solares emitidas en ambos periodos de tiempo.

Las series mensuales de precipitaciones fueron representadas por modelos multiplicativos y las de las series anuales por modelos aditivos. En tanto que la cantidad de manchas solares en ambos momentos (mensual y temporal) por modelos aditivos.

Los errores en todos los modelos no son independientes, sino que presentan una estructura de correlación temporal, la cual fue capturada a través del variograma. En el caso de las series mensuales el variograma ajustado corresponde a una función de la familia exponencial para todas las series. Para las series anuales, el variograma que modela la estructura de correlación temporal en el caso de la precipitación proviene de la familia exponencial para la ciudad de Río Gallegos y de la familia esférica para la ciudad de Punta Arenas, y para la cantidad de manchas solares de la familia esférica.

Los datos de precipitación media mensual entre ambas ciudades presentan correlación (pvalor $=0.000$ ), aunque dicha evidencia es mucho más débil si se consideran las series de precipitaciones media anuales.

Por otro lado, luego de plantear distintas hipótesis sobre la posible influencia de la cantidad de manchas solares emitidas sobre las precipitaciones se puede asegurar que no existe influencia 
del número de manchas solares mensuales sobre las precipitaciones para ninguna de las dos ciudades en el periodo en curso tanto para los datos mensuales ( $\mathrm{p}-\mathrm{v}^{\mathrm{alo}} \mathrm{r}_{\mathrm{P}}=0.8231 \mathrm{y} \mathrm{p}$ valor $_{S}=0.9429, \mathrm{p}$-valor $\mathrm{P}=0.2739 \mathrm{y}$ - valor $_{\mathrm{S}}=0.8902$ para los coeficiente de correlación de Pearson, denotado como p-valor ${ }_{\mathrm{P}}$, y Spearman, representado por $\mathrm{p}$-valor $\mathrm{S}_{\mathrm{S}}$, con datos mensuales de Río Gallegos y de Punta Arenas respectivamente), como para las series anuales

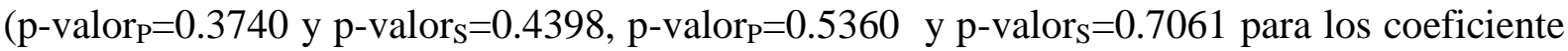
de correlación de Pearson y Spearman con datos anuales de Río Gallegos y de Punta Arenas respectivamente).

Este mismo comportamiento se da para el número de manchas solares y las series de precipitaciones medias mensuales y anuales pero desplazadas en el tiempo (en este caso todas las correlaciones dan no significativas nuevamente).

Así, a diferencia de lo acontecido en otras regiones del planeta en donde la cantidad de manchas solares influye en las precipitaciones, podemos asegurar que para la región ubicada en el rectángulo definido por los $51^{\circ}$ y $55^{\circ}$ de latitudes sur, y entre los $69^{\circ}$ y $71^{\circ}$ de longitud oeste, en donde se encuentran emplazadas Río Gallegos y Punta Arenas, no existe evidencia de la influencia de las manchas solares sobre la precipitación para las series anuales y mensuales.

\section{REFERENCIAS}

ABBOT, C. G. (1966). Solar Variation, a Weather Element. Proceedings of the National Academy of Sciences of the United States of America, 56(6), 1627-1634. https://doi.org/10.1073/pnas.56.6.1627

BAL, S., BOSE, M. (2010). A climatological study of the relations among solar activity, galactic cosmic ray and precipitation on various regions over the globe. Journal Earth Systems Science, 119 (2): 201-209. https://doi.org/10.1007/s12040-010-0015-8

ARENAS VAZQUEZ, F., ALIAGA BUSTOS, G., MARCHANT SANTIAGO, C., SANCHEZ ACUÑA, R. (2010). El espacio geográfico Magallánico: Antecedentes acerca de su estructura y funcionamiento. Universidad del Bio Bio Revista Tiempo y Espacio, 25. ISSN 0716-9671.

BALDWIN, M., DUNKERTON, T. J., (2005). The solar cycle and stratosphere-troposphere dynamical coupling. Journal of Atmospheric and Solar-Terrestrial Physics, 67: 71-82. https://doi.org/10.1016/j.jastp.2004.07.018

BONFILI, O., SOTO, J., MAGLIONE, D. (2016). Características Generales de los Principales Elementos Climáticos de la Ciudad de Río Gallegos, Congreso Internacional de Geografía - $77^{\circ}$ semana de geografía, San Miguel de Tucumán.

CARSLAW, K.S., HARRISON, R.G., KIRKBY, J., (2002). Cosmic Rays Clouds and Climate. Science, 298: 1732-1738. https://doi.org/10.1126/science.1076964

CLAVET, X., ROMERO, M. C., SANCHO, J.M, RIPODAS, P., QUINTERO, V.J., (2001). Relationship between sunspot number and total annual precipitation at Iza-a (Tenerife): Maximum precipitation prediction with three year lagged sunspots?. ArXiv Physics e-prints. Recuperado eprintarXiv:physics/0110083.

HARRISON, R.G., STEPHESON, D.B., (2006). Empirical evidence for a nonlinear effect of galactic cosmic rays on clouds. Proceedings of the Royal Society, 462: 1221-1233. https://doi.org/10.1098/rspa.2005.1628 
HATHAWAY, D. H., WILSON, R. M., (2004). What the sunspot record tells us about space climate. Solar Physics, 224: 5-19. https://doi.org/10.1007/s11207-005-3996-8

HELD, I. M. (2012). Issues in Climate Science Underlying Sun/Climate Research. National Research Council. Committee on the Effects of Solar Variability on Earth's Climate. Space Studies Board. Division on Engineering and Physical Sciences. The effects of solar variability on earth's climate A workshop report. The National Academies Press. Washington, D.C., p. 21.

HIREMATH, K.M., MANJUNATH, H., SOON, W. (2015). Indian summer monsoon rainfall: Dancing with the tunes of the sun. New Astronomy, 35: 8-19. https://doi.org/10.1016/j.newast.2014.08.002

JIMENEZ, J.A.G. (2012). Anillos, clima y actividad solar. Universidad Nacional de Colombia. Tesis para Maestria. Facultad de Ciencias Agrarias, Departamento de Ciencias Forestales.

KASATKINA, E., SHUMILOV, O., LUKINA, N.V., KRAPIEC, M., JACOBY, G. (2007). Stardust component in tree rings. Dendrochronologia, 24: 131-135. https://doi.org/10.1016/j.dendro.2006.10.005

KNIVETON, D.R. (2004). Precipitation, cloud cover and Forbush decreases in galactic cosmic rays. Journal of Atmospheric and Solar Terrestrial Physics, 66: 1135-1142 https://doi.org/10.1016/j.jastp.2004.05.010

LANE, L. J., NICHOLSs, M. H., OSBORN, H. B. (1994). Time series analyses of global change data. Environ. Pollut, 83: 63-68. https://doi.org/10.1016/0269-7491(94)90023$\underline{X}$

LEAN, J., BEER, J., BRADLEY, R. (1995). Reconstruction of solar irradiance since 1610: Implications for climate change. Geophysical Research Letters. 22: 3195-3198. https://doi.org/10.1029/95GL03093

LEGRAS, B., MESTRE, O., BARD, E., YIOU, P. (2010). A critical look at solar-climate relationships from long temperature series.Clim. Past., 6: 745-758, doi:10.5194/cp-6745-2010. https://doi.org/10.5194/cp-6-745-2010

MAGLIONE, D., SÁENZ, J. L., SOTO, J., BONFILI, O., TALAY, C., SANDOVAL, M., LLANCALAHUEN, M. (2014). Patrones de Comportamiento de Elementos Meteorologicos en Patagonia Sur. Bienal del III Encuentro de Investigadores de la Patagonia Austral - $3^{\circ}$ Edición (ISBN 978-987-3714-00-9). Río Turbio, Argentina.

MAUAS, P.J.D., BUCCINO, A.P., FLAMENCO, E. (2010). Long-term solar activity influences on South American rivers. Journal of Atmospheric and Solar-Terrestrial Physics, 73: 377-382. Medellín, Colombia. Cap. II, pp. 39-59.

NAVARRO, M., VILATTE, C., AGUAS, L. (2013). NOAA.The Sunspot Cycle. Boletín Agrometeorológico del Centro Sur de la Provincia de Buenos Aires. Centro Regional de Agrometeorología - U.N.C.P.B.A. v. 1 al 18. Recuperado de http://solarscience.msfc.nasa.gov/SunspotCycle.shtml

MEEHL, G. A. y TENG, H. (2012). Case studies for initialized decadal hindcasts and predictions for the Pacific region. Geophys. Res. Lett. 39, L22705. https://doi.org/10.1029/2012GL053423

NATIONAL RESEARCH COUNCIL (2012). The Effects of Solar Variability on Earth's Climate: A Workshop Report. Washington, DC: The National Academies Press. 
OESCHGER, H., BEER, J., ANDREE, M. (1987). 10Be and 14C in the earth system Philosophycal Transactions of the Royal Society of London Series A Mathematical and Physics Sciences, 323: 45-55.

PALLÉ, E., BUTLER, C., O' BRIEN, K. (2004). The possible connection between ionization in the atmosphere by cosmic rays and low-level clouds. Journal of Atmospheric and Solar-TerrestrialPhysics, 66 https://doi.org/10.1016/i.jastp.2004.07.041

PEDREROS, M. (2013). Manchas en el sol: ¿lluvias en la tierra?. Publicaciones del Departamento de Física, Universidad de Tarapacá. Vol.17. Recuperado de http://www.uta.cl/charlas/volumen17/Indice/MPedreros.pdf

RASPOPOV, O.M, DERGACHEVB, V.A., KOLSTRO, T., 2004. Periodicity of climate conditions and solar variability derived from dendrochronological and other palaeoclimatic data in high latitudes. Palaeogeography, Palaeoclimatology, Palaeoecolgy, 209: 127-139. https://doi.org/10.1016/j.palaeo.2004.02.022

ROMEU I CODINA E., y LÓPEZ BUSTIN J.A. (2006). Aproximación a la posible existencia de la se-al de los ciclos solares 18 y 19 en la pluviometría de la península Ibérica. Gepgraphicalia, 49: 19-36.

RONTU CARLON, N., PAPANASTAIOU, D. K., FLEMING, E. L., JACKMAN, C. H., NEWMAN, P. A., BURKHOLDER, J. B. (2010). UV absorption cross sections of nitrous oxide (N2O) and carbon tetrachloride (CCl4) between 210 and $350 \mathrm{~K}$ and the atmospheric implications. Atmos. Chem. Phys., 10: 6137-6149. https://doi.org/10.5194/acp-10-6137-2010

SELAVARAJ, R. S., ADITA, R. (2012). The solar influence on the monsoon rainfall over Tamil Nadu. J. Ind. Geophys. Union, 16(3): 107-111.

STAGER, J. C., RUZMAIKIN, A., CONWAY, D., VERBURG, P., MASON, P. J. (2007). Sunspots, El Ni-o, and the levels of Lake Victoria, East Africa. Journal Geophysical Research, 112, D15106. https://doi.org/10.1029/2006JD008362

SVENSMARK, H. (1998). Influence of Cosmic Rays on Earth's Climate, Physical Review Letters, 81: 5027-5030. https://doi.org/10.1103/PhysRevLett.81.5027

SVENSMARK, H., BONDO, T., SVENSMARK, J. (2009). Cosmic rays decreases affect atmospheric aerosols and clouds. GeophysicalResearhLetters, 36: L15101, doi: 10.1029/2009GL038429. https://doi.org/10.1029/2009GL038429

SVENSMARK, H., FRIIS-CHRISTENSEN, E. (1997). Variation of cosmic ray flux and global coverage - a missing link in solar climate relationships. Journal of Atmospheric and solar Terrestrial Physics, 59: 1225-1232. https://doi.org/10.1016/S13646826(97)00001-1

TODD, M. C., KNIVETON, D. R. (2004). Short-term variability in satellite derived cloud cover and galactic cosmic rays: an update, J. Atmos. Sol.-Terr. Phy., 66: 1205-1211. https://doi.org/10.1016/j.jastp.2004.05.002

TINSLEY, B. A., YU, F. (2004). Atmospheric Ionization and Clouds as Links Between Solar Activity and Climate, in Pap, Judit M.; Fox, Peter, Solar Variability and its Effects on Climate, 141, American Geophysical Union, 321-339. https://doi.org/10.1029/141GM22

USOSKINn, I.G., SOLANKI, S.K., SCHÜSSLER, M., MURSULA, K., ALANKO, K. (2003). Millenium-Scale sunspot number reconstruction: evidence for an unusually active sun since 1940s. The American Physical Society, 91: 1-4. 
YEPES, A., BUKERIDGE, M. (2011). Respuestas de las plantas ante los factores ambientales del cambio climático global (Revisión). Colombia Forestal, 14: 213-232. https://doi.org/10.14483/udistrital.jour.colomb.for.2011.2.a06

ZHAO, L., WANG, J., ZHAO, H. (2012). Solar Cycle Signature in Decadal Variability of Monsoon Precipitation in China. Journal of the Meteorological Society of Japan, 90(1): 1-9. https://doi.org/10.2151/jmsj.2012-101 